\title{
Safety and effectiveness of classical and alternative sunitinib dosing schedules for metastatic renal cell carcinoma: a meta-analysis
}

Seye Abogunrin*,1, Ajibade O Ashaye², Joseph C Cappelleri ${ }^{3}$, Andrew G Clair ${ }^{4}$, Kyle Fahrbach ${ }^{5}$, Krishnan Ramaswamy ${ }^{4}$, Lucile Serfass ${ }^{6}$, Sandy Srinivas ${ }^{7}$, Despina Thomaidou ${ }^{8}$ \& Giovanni Zanotti ${ }^{4}$

${ }^{1}$ Meta Research, Evidera, London, W6 8BJ, UK

${ }^{2}$ Formerly Meta Research, Evidera, Waltham, MA 02451, USA

${ }^{3}$ Pfizer Inc, Groton, CT 06340, USA

${ }^{4}$ Pfizer, Inc., New York, NY 10017, USA

${ }^{5}$ Meta Research, Evidera, Waltham, MA 02451, USA

${ }^{6}$ Pfizer Oncology, 75668 Paris, France

${ }^{7}$ Stanford University Medical Center, Stanford, CA 94304, USA

${ }^{8}$ Pfizer, 15451 Athens, Greece

*Author for correspondence: Tel.: +44 (0)208 576 5049; seye.abogunrin@evidera.com

The optimal dosing schedule to maintain the effectiveness of sunitinib for metastatic renal cell carcinoma - while reducing toxicity - remains an important clinical question. A meta-analysis of randomized trials and observational studies assessed the relative treatment effects of $4 / 2,2 / 1$ and transitional-2/1 schedules on outcomes and adverse events using Bayesian network meta-analysis methods. Treatment with 2/1 reduced the risk of disease progression or death by $25 \%$ and had lower odds of hand-and-foot syndrome compared with the 4/2. A numerical but not 'statistical' benefit in progression-free survival was observed with the transitional-2/1 compared with 4/2. Alternative schedules with the 2/1 and transitional-2/1 may be more clinically beneficial in metastatic renal cell carcinoma than the $4 / 2$ schedule.

First draft submitted: 1 November 2018; Accepted for publication: 8 April 2019; Published online: 23 April 2019

Keywords: alternative dosing schedule $\bullet$ metastatic renal cell carcinoma $\bullet$ sunitinib $\bullet$ transitional dosing schedule

Over 270,000 new cases of renal cell carcinoma (RCC) are diagnosed every year accounting for about $90 \%$ of kidney cancers $[1,2]$. The economic impact of treating metastatic clear cell renal cell carcinoma (mRCC), which constitutes about one in every three RCC cases at diagnosis, is estimated to be approximately US $\$ 1.6$ billion in some selected countries [3]. The introduction of target agents, mainly tyrosine kinase inhibitors (TKIs) of VEGF receptors, have improved outcomes for patients with mRCC $[4,5]$ in the last decade. The recent introduction of novel immunotherapeutic agents has provided important additional benefits [6-9]. However, VEGFR TKIs currently remain a mainstay of RCC treatment, and continue to play an important role in the management of treatment-naive and pretreated patients.

Sunitinib is an oral, multitargeted TKI with antiangiogenic and antitumor activity. A pivotal Phase III study of treatment-naive patients with mRCC determined sunitinib prolonged median progression-free survival (PFS) and objective response rate (ORR) compared with IFN- $\alpha$. It also extended median overall survival (OS) beyond 2 years for the first time in the history of mRCC $[10,11]$. Based on these results, sunitinib has become one of the standards of care for treatment-naive patients with mRCC, and is currently the most commonly used agent in this setting [12].

Sunitinib is administered to patients with mRCC on a schedule of 4 weeks on with a 2-week break before the next dose (hereafter referred to as 'classical $4 / 2$ schedule'). This 2 -week break is intended for patients' recovery from known toxicities of the conventional 4-week daily dose. The classical 4/2 schedule has been shown to cause high incidences of adverse events (AEs) in Phase III clinical trials and clinical practice, including fatigue,

Future $\because$ Medicine 
diarrhea, stomatitis, hematological toxicity and hand-foot syndrome. Consequently, its use frequently requires dose reductions and/or treatment interruptions or discontinuation [11,13-15].

Unnecessary dose reductions and/or treatment interruptions could result in the lowering of dose intensity and drug exposure, which may compromise efficacy [16]. Considering these risks, alternative treatment schedules have been explored in real-world practice including a 2-week on with a 1-week break (hereafter referred to as 'alternative $2 / 1$ schedule') schedule and a transition from the classical schedule to the alternative schedule (hereafter referred to as 'transitional $2 / 1$ schedule') in patients experiencing relevant toxicities. It is commonly known that sunitinib is most effective when given to patients with mRCC at the full dosage of $50 \mathrm{mg}$ daily [16], but the optimal schedule to maintain its demonstrated activity, while minimizing toxicity is not yet established, despite several studies evaluating the role of alternative sunitinib schedules [17-20]. Therefore, we conducted a systematic literature review (SLR) and meta-analysis (MA) to assess the safety and effectiveness of the classical $4 / 2$, alternative $2 / 1$ and transitional $2 / 1$ sunitinib dosing schedules, to provide treatment recommendations in patients with mRCC who are or may be unable to tolerate the classical $4 / 2$ schedule.

\section{Methods}

Systematic literature review

The SLR was conducted according to Preferred Reporting Items for Systematic Reviews and Meta-Analyses (PRISMA) guidelines [21]. A protocol was developed for this SLR; however, it was not preregistered or registered publicly.

\section{Identification \& selection of studies}

The Cochrane Collaboration Central Register of Controlled Trials, Embase and MEDLINE were searched to identify studies published up to November 2017 using keywords synonymous with 'renal cell carcinoma' and 'sunitinib'. The American Society of Clinical Oncology (ASCO), the ASCO Genitourinary Cancers Symposium (GU) and bibliographies of SLRs were also searched, with, to identify other publications of potential relevance. Studies were eligible if they were Phase II and III randomized controlled trials (RCTs) or longitudinal observational studies with a comparative design. They were also eligible if the studies reported data on safety and effectiveness outcomes in patients with mRCC treated with the classical $4 / 2$ or any alternative $2 / 1$ dosing schedule, and if they were published in English. The search strategies and prespecified study selection criteria can be found in the Supplementary Information (Supplementary Tables 1-5).

The study selection criteria were used by two reviewers to screen the titles, abstracts and full texts for relevance to the SLR. Any discrepancies were resolved by a third reviewer.

\section{Data extraction}

For each study included in the review, details of the study design, patient characteristics, interventions and outcomes were extracted by one reviewer and quality checked for accuracy and correctness by another reviewer.

\section{Meta-analyses}

Feasibility assessment

A feasibility assessment was conducted, prior to conducting the MA on the included studies, to determine whether there was sufficient, available data amenable for analysis and exploration of potential sources of heterogeneity within the studies. The viability of creating a network of included studies was assessed by determining whether the included studies were comparable with regards to study setting, design, treatment, patient characteristics and outcome definitions. Observational studies considered in the feasibility assessment were also qualitatively assessed for sampling bias by checking for the presence of patient channeling, which we defined as prescribing an intervention to a preferred group of patients that will likely benefit from that treatment [22].

\section{Meta-analytical methods}

Mixed treatment comparisons [23,24] of the three dosing schedules, identified as classical $4 / 2$, transitional $2 / 1$ or alternative 2/1, were conducted using Bayesian methods to obtain relative treatment effects. This involved indirect and pairwise MAs as well as network meta-analyses (NMAs) of all outcomes that were deemed analyzable. The results were presented for both sets of analyses. NMAs are typically conducted on randomized data, but an NMA was performed on non-randomized data to compare the classical $4 / 2$ schedule with the transitional $2 / 1$ schedule 
because data from RCTs were unavailable. Random- and fixed-effects models were employed when appropriate, and analyses were limited to fixed-effects models in cases where there was limited information to estimate a random-effects variance. Otherwise, results of random-effects models were presented.

Statistical heterogeneity was quantified using the $I^{2}$ statistic [25]. Two sensitivity analyses that excluded outlier data were conducted for each of the time-to-event effectiveness outcomes. The first sensitivity analysis excluded data for OS and PFS reported for any dosing schedules from the only RCT identified by the SLR - Lee et al. [26]. The second used data, also for OS and PFS, from all patients randomized to the classical 4/2 schedule arm of the RCT.

The relative effects for OS and PFS were estimated with hazard ratios (HRs) and their associated $95 \%$ credible intervals (CrIs). Time-to-treatment progression (TTP) data were used as proxy in one instance for PFS due to a lack of data [26]. ORR, partial response (PR), complete response (CR), stable disease (SD), progressive disease (PD) and grade 3-4 AEs were assessed and presented as odds ratios (ORs) and their associated 95\% CrIs. A statistical advantage was demonstrated if the CrIs did not cross 1 , while trends for the treatment and comparator arms were demonstrated when CrIs crossed 1 but HRs or ORs were $<0.80$ and $>1.25$, respectively.

All analyses were conducted using OpenBugs 3.2.2.

\section{Results}

\section{Systematic literature review}

Of 692 unique records reviewed, full texts of 11 primary studies (12 publications) met the SLR protocol's prespecified inclusion criteria. No additional publications were identified from the conference or bibliography searches (Supplementary Figure 1 in Supplementary Appendix). Most studies were of retrospective cohort design with data sourced from medical records. Only two clinical trials were identified, one comparative, nonrandomized Phase II trial [27] and one multicenter, Phase II RCT [26]. Study samples ranged from 30 to 460 patients across four continents. Four studies each were identified for Asia and North America, while one study each reported data on populations from Africa and Europe. The last study did not provide details of the geographic location.

\section{Meta-analysis}

Feasibility assessment

Nine [26-35] of the 11 studies provided evidence on safety and effectiveness outcomes that was deemed eligible for comparison via NMA (Table 1; Figure 1). Meta-analyzable effectiveness outcomes were OS, PFS, ORR, CR, PR, SD and PD. Safety outcomes contributing to the NMA were grade 3-4 AEs inclusive of fatigue, nausea, diarrhea, thrombocytopenia, leukopenia, anemia, neutropenia and hand-foot syndrome. The populations were broadly similar across the nine studies in terms of age, gender, Eastern Cooperative Oncology Group (ECOG) status and Memorial Sloan Kettering Cancer Center (MSKCC) risk group. Patient recruitment periods spanned a decade from October 2004 to September 2014.

Two observational studies were considered ineligible for the MA as they did not compare at least two of the dosing schedules of interest. While both studies included patients who had received the classical $4 / 2$ schedule, one included patients who received a variety of alternative schedules - namely $2 / 1(82 \%), 7$ days on and 3 days off alternating with 7 days on and 4 days off (8\%) and another alternative schedule (details not reported; 9\%) [17] - and the other included patients who received continuous daily dosing as well as other alternative schedules, specific details of which were not reported [33]. Table 1 provides an overview of the dosing schedules evaluated in the included studies.

Definitions of OS were similar across four studies (i.e., the time between enrollment and death from any cause). PFS was defined as time from enrollment to the occurrence of disease progression or death from any cause in the six studies when this outcome was assessed. Lee et al. [26] did not report data on PFS. TTP was considered the most similar outcome and was therefore used as a proxy for PFS.

\section{MA results: alternative $2 / 1$ versus classical $4 / 2$ schedule}

Effectiveness

Treatment with the alternative $2 / 1$ schedule prolonged PFS in the base-case analysis compared with treatment with the classical 4/2 schedule (HR: 0.75 [95\% CrI: 0.60-0.94]). Additional sensitivity analyses did not find any statistical advantages for either schedule. No 'statistical differences' were found in OS between the dosing schedules observed in base-case or sensitivity analyses. There were also no statistical differences observed between dosing 


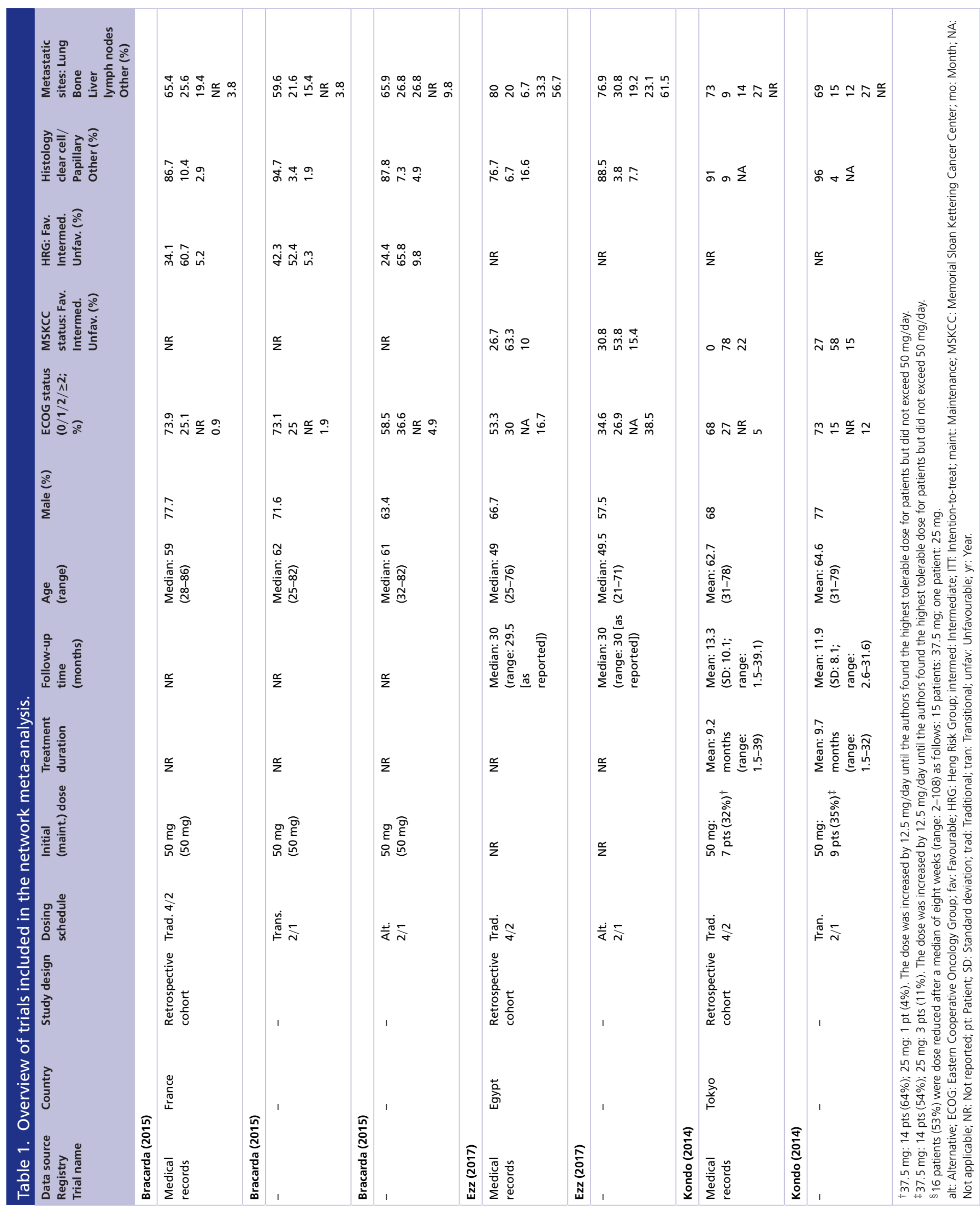




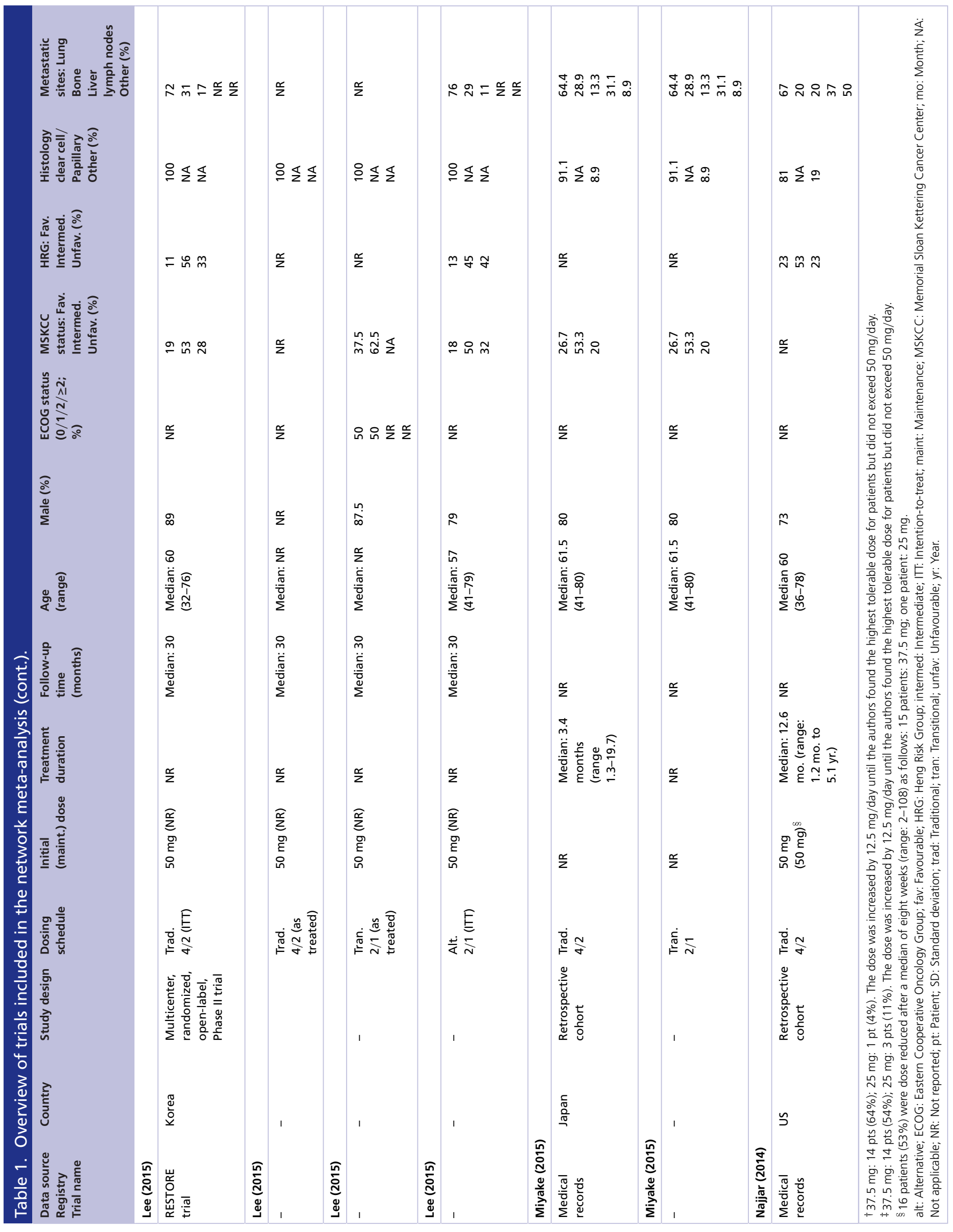




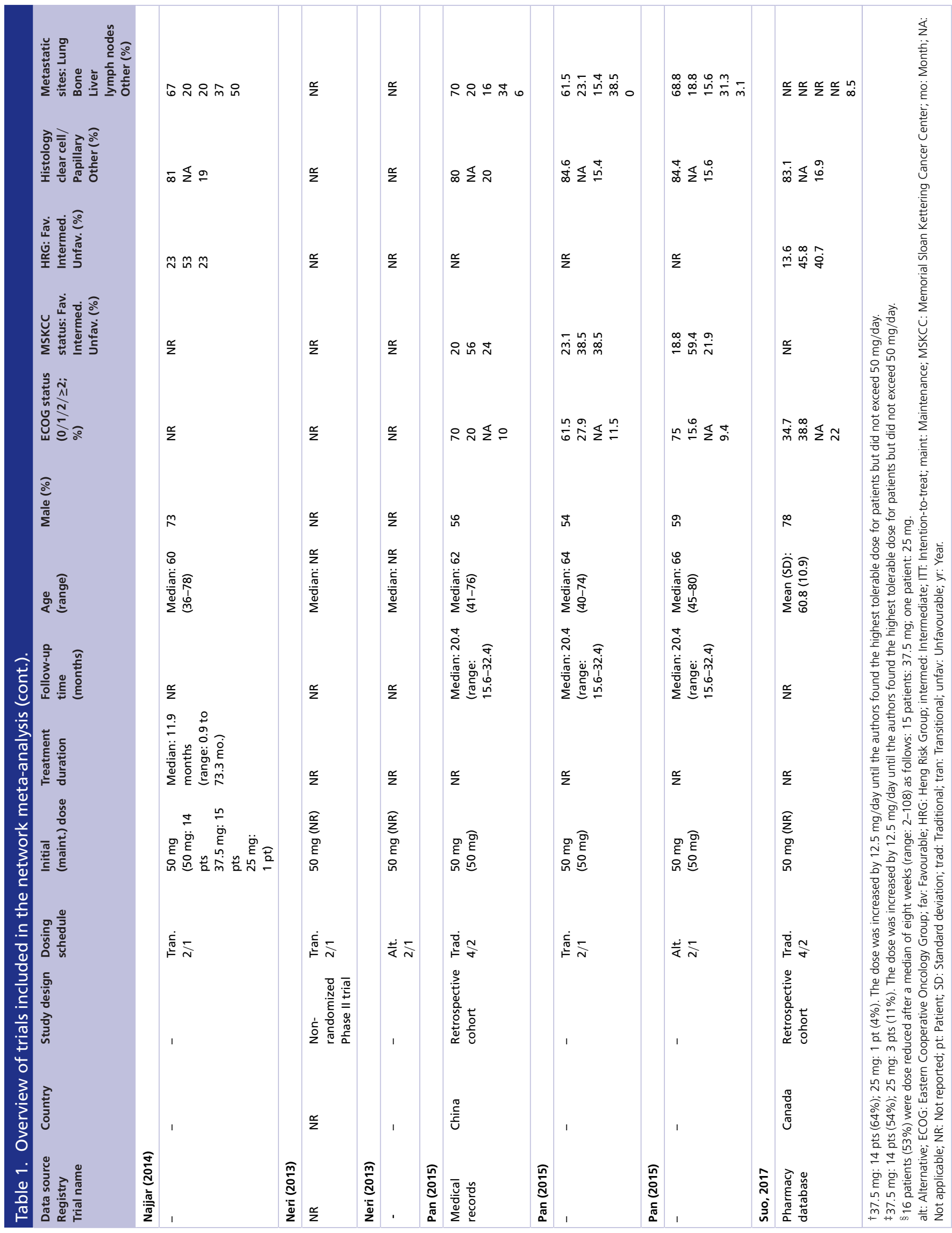




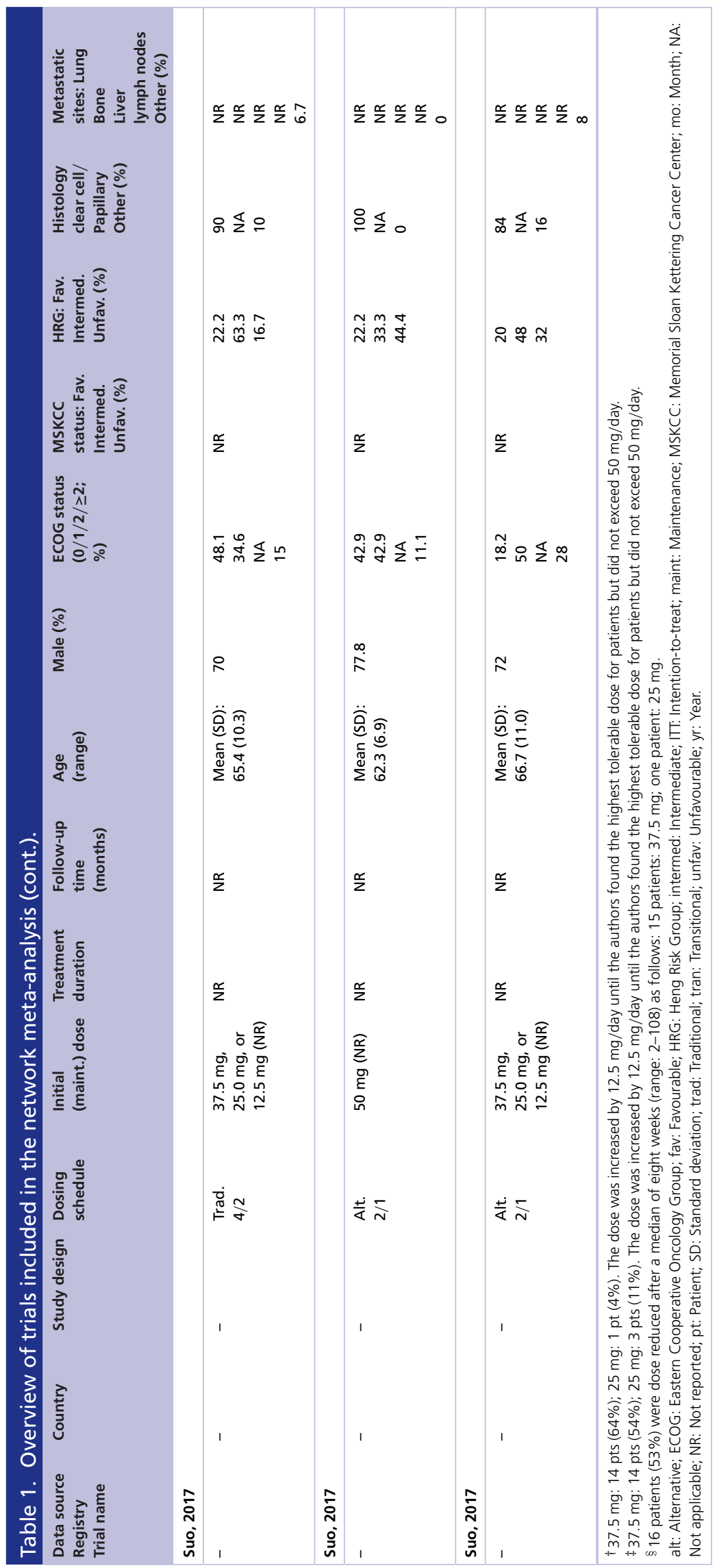




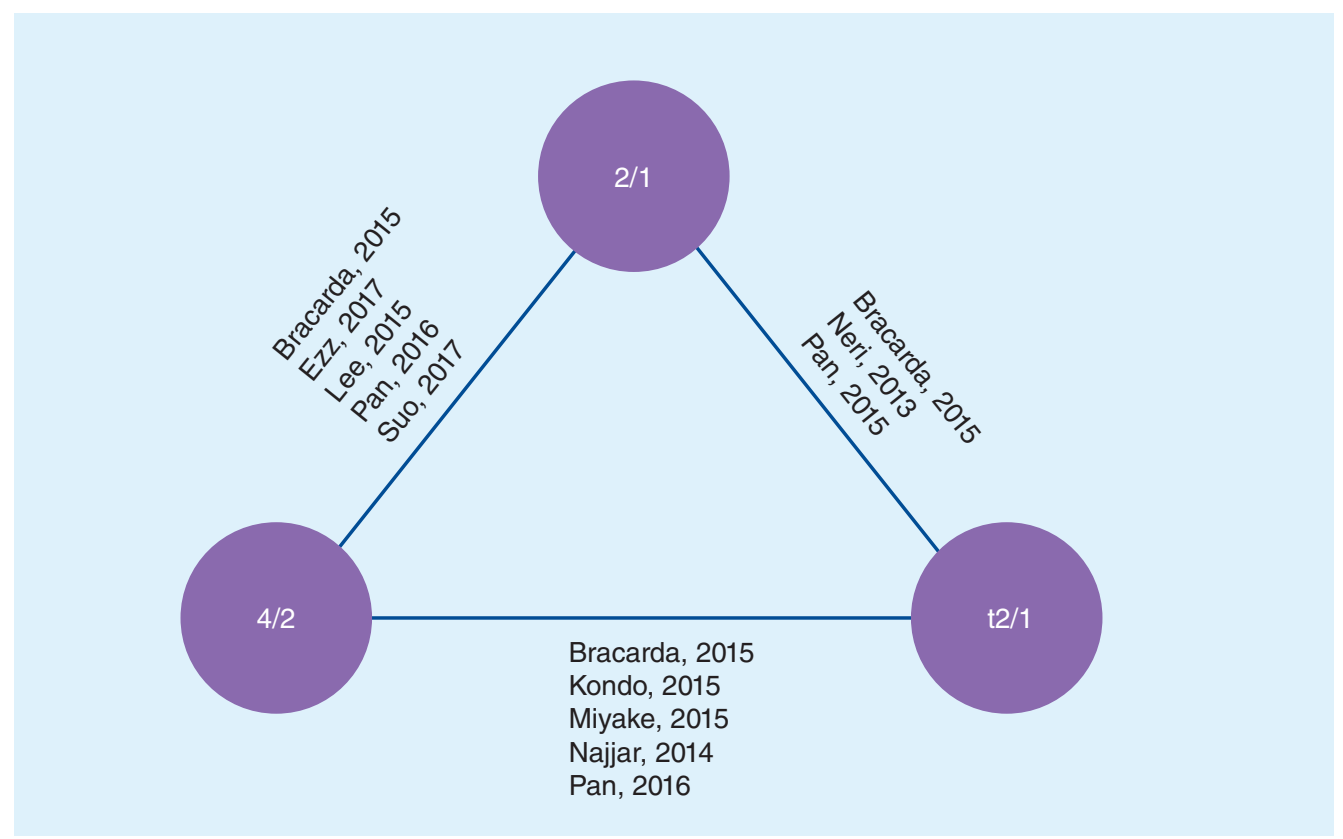

Figure 1. Network diagram.

2/1: Alternative 2/1 schedule; 4/2: Classical 4/2 schedule; t2/1: Transitional schedule (4/2 switch to 2/1).

schedules for CR, ORR, PR, PD or SD. The MA results for OS and PFS are presented in Table 2. MA results for response outcomes appear in Table 3.

Safety

Patients treated with the classical $4 / 2$ schedule had substantially higher odds of having grade $3-4$ hand-foot syndrome compared with those treated with the alternative 2/1 schedule (OR: 0.33 [95\% CrI: 0.12-0.79]). Although the classical $4 / 2$ schedule was associated with numerically higher odds of fatigue, neutropenia and thrombocytopenia, no statistical differences between these dosing schedules were observed for any other grade 3-4 events. The results for the alternative $2 / 1$ schedule compared with the classical $4 / 2$ schedule with regards to anemia and diarrhea were similar (anemia OR: 0.97 [95\% CrI: 0.33-2.89] and diarrhea OR: 0.99 [95\% CrI: 0.26-3.7]). The MA results for safety outcomes are presented in Table 4 and Table 5.

\section{MA results: transitional $2 / 1$ versus classical 4/2 schedule}

Effectiveness

Treatment with the transitional 2/1 schedule showed a trend for prolonging PFS compared with treatment with the classical 4/2 schedule (HR: 0.4 [95\% CrI: 0.14-1.12]). The network for PFS included only three studies, two of which reported conflicting directionality of PFS outcomes (PFS and TTP). The sensitivity analysis that excluded RCT data further weakened the base-case findings as demonstrated through wider confidence intervals. No statistical differences were observed between dosing schedules for CR, ORR, PR, PD or SD. However, there was a decreased trend for patients who were treated with the transitional 2/1 schedule to achieve CR (OR: 1.29 [95\% CrI: 0.23-7.36]), and an increased trend of achieving PR (OR: 0.74 [95\% CrI: 0.17-3.25]) and ORR (OR: 0.79 [95\% CrI: 0.25-2.53]) compared with those treated with the classical $4 / 2$ schedule.

Safety

Patients treated with the transitional $2 / 1$ schedule, compared with the classical $4 / 2$ schedule, had statistically lower odds of having grade 3-4 diarrhea (OR: 0.32 [95\% CrI: 0.12-0.87]), fatigue (OR: 0.34 [95\% CrI: 0.15-0.75]) and hand-foot syndrome (OR: 0.37 [95\% CrI: 0.18-0.75]). There were no statistical differences observed between these dosing schedules for any other grade 3-4 AEs. However, patients on the transitional 2/1 schedule had numerically lower odds of having leukopenia (OR: 0.72 [95\% CrI: 0.13-4.11]), nausea (OR: 0.4 [95\% CrI: 0.07-2.1]) and 
Table 2. Summary of pairwise meta-analysis and network meta-analysis effectiveness results: survival outcomes.

\begin{tabular}{|c|c|c|c|c|c|c|c|c|c|c|c|}
\hline \multirow{3}{*}{$\begin{array}{l}\text { Outcome } \\
\text { Model }\end{array}$} & \multicolumn{6}{|c|}{ PFS } & \multicolumn{5}{|c|}{ os } \\
\hline & \multicolumn{2}{|c|}{$\begin{array}{l}\text { Base case } \\
\text { (all studies) }\end{array}$} & \multicolumn{2}{|c|}{$\begin{array}{l}\text { Sensitivity analyses } \\
\text { (without Lee, 2015) }\end{array}$} & \multicolumn{2}{|c|}{$\begin{array}{l}\text { Sensitivity analyses (with } \\
\text { ITT from Lee, 2015) }\end{array}$} & \multicolumn{2}{|c|}{$\begin{array}{l}\text { Base case } \\
\text { (all studies) }\end{array}$} & \multirow{2}{*}{$\begin{array}{l}\text { Sensitivity } \\
\text { analyses } \\
\text { (without } \\
\text { Lee, 2015) } \\
\text { FE }\end{array}$} & \multicolumn{2}{|c|}{$\begin{array}{l}\text { Sensitivity analyses (with } \\
\text { ITT from Lee, 2015) }\end{array}$} \\
\hline & FE & $\mathrm{RE}$ & FE & RE & $\mathrm{FE}$ & RE & FE & RE & & $\mathrm{FE}$ & RE \\
\hline Comparisons & HR ( $95 \% \mathrm{Crl})$ & & & & & & & & & & \\
\hline $\begin{array}{l}\text { Alternative } \\
2 / 1 \text { vs } \\
\text { classical } 4 / 2 \\
\text { (NMA) }\end{array}$ & $\begin{array}{l}0.75 \\
(0.6-0.94)^{\dagger}\end{array}$ & $\begin{array}{l}0.74 \\
(0.45-1.21)\end{array}$ & $\begin{array}{l}0.81 \\
(0.63-1.03)\end{array}$ & $\begin{array}{l}0.79 \\
(0.45-1.4)\end{array}$ & $0.8(0.64-1)$ & $\begin{array}{l}0.8 \\
(0.61-1.03)\end{array}$ & $\begin{array}{l}0.98 \\
(0.75-1.29)\end{array}$ & $\begin{array}{l}0.97 \\
(0.62-1.5)\end{array}$ & $\begin{array}{l}0.94 \\
(0.69-1.28)\end{array}$ & $\begin{array}{l}0.98 \\
(0.75-1.29)\end{array}$ & $\begin{array}{l}0.97 \\
(0.62-1.48)\end{array}$ \\
\hline $\begin{array}{l}\text { Alternative } \\
2 / 1 \text { vs } \\
\text { classical } 4 / 2 \\
\text { (pairwise } \\
\text { MA) }\end{array}$ & $\begin{array}{l}0.75 \\
(0.6-0.94)^{\dagger}\end{array}$ & $\begin{array}{l}0.75 \\
(0.6-0.94)^{\dagger}\end{array}$ & $\begin{array}{l}0.81 \\
(0.64-1.03)\end{array}$ & $\begin{array}{l}0.81 \\
(0.64-1.03)\end{array}$ & $0.8(0.64-1)$ & $0.8(0.64-1)$ & $\begin{array}{l}0.98 \\
(0.75-1.29)\end{array}$ & $\begin{array}{l}0.98 \\
(0.74-1.3)\end{array}$ & $\begin{array}{l}0.94 \\
(0.69-1.27)\end{array}$ & $\begin{array}{l}0.98 \\
(0.75-1.29)\end{array}$ & $\begin{array}{l}0.98 \\
(0.74-1.3)\end{array}$ \\
\hline $\begin{array}{l}\text { Transitional } \\
2 / 1 \text { vs } \\
\text { classical } 4 / 2 \\
\text { (NMA) }\end{array}$ & $\begin{array}{l}0.28 \\
(0.24-0.33)^{\dagger}\end{array}$ & $\begin{array}{l}0.41 \\
(0.21-0.84)^{\dagger}\end{array}$ & $\begin{array}{l}0.29 \\
(0.24-0.35)^{\dagger}\end{array}$ & $\begin{array}{l}0.49 \\
(0.22-1.19)\end{array}$ & NA & NA & $\begin{array}{l}0.33 \\
(0.25-0.43)^{\dagger}\end{array}$ & $\begin{array}{l}0.33 \\
(0.15-0.7)^{\dagger}\end{array}$ & $\begin{array}{l}0.32 \\
(0.25-0.42)^{\dagger}\end{array}$ & NA & NA \\
\hline $\begin{array}{l}\text { Transitional } \\
2 / 1 \text { vs } \\
\text { classical } 4 / 2 \\
\text { (pairwise } \\
\text { MA) }\end{array}$ & $\begin{array}{l}0.33 \\
(0.26-0.42)^{\dagger}\end{array}$ & $\begin{array}{l}0.4 \\
(0.14-1.12)\end{array}$ & $\begin{array}{l}0.34 \\
(0.27-0.43)\end{array}$ & $\begin{array}{l}0.53 \\
(0.13-2.22)\end{array}$ & NA & NA & NA & NA & NA & NA & NA \\
\hline Sqrt(tau) & NA & 0.5303 & NA & 0.5701 & NA & 0.1064 & NA & 0.2457 & NA & NA & 0.2438 \\
\hline DIC & 33.39 & 10.09 & 30.95 & 7.117 & -0.3935 & 1.481 & 3.412 & 4.541 & 3.648 & 3.876 & 4.971 \\
\hline
\end{tabular}

† Statistically significant results. heterogeneity parameter, which indicates the variance of the underlying distribution.

Crl: Credible interval; DIC: Deviance information criterion; FE: Fixed-effects; HR: Hazard ratio; ITT: Intention-to-treat; MA: Meta-analysis; N/A: Not applicable; NMA: Network meta-analysis; OR: Odds ratio; OS: Overall survival; PFS: Progression-free survival; RE: Random-effects.

Table 3. Summary of pairwise meta-analysis and network meta-analysis effectiveness results: response outcomes.

\begin{tabular}{|c|c|c|c|c|c|c|c|c|c|c|}
\hline \multirow{3}{*}{$\begin{array}{l}\text { Outcome } \\
\text { Model }\end{array}$} & \multicolumn{2}{|c|}{ Objective response } & \multicolumn{2}{|c|}{ Complete response } & \multicolumn{2}{|c|}{ Partial response } & \multicolumn{2}{|c|}{ Progressive disease } & \multicolumn{2}{|c|}{ Stable disease } \\
\hline & \multicolumn{10}{|c|}{ Base case } \\
\hline & FE & RE & FE & RE & FE & RE & $\mathrm{FE}$ & RE & FE & RE \\
\hline Comparisons & \multicolumn{10}{|l|}{ OR (95\% Crl) } \\
\hline $\begin{array}{l}\text { Alternative } \\
2 / 1 \text { vs } \\
\text { classical } 4 / 2 \\
\text { (NMA) }\end{array}$ & $\begin{array}{l}1.22 \\
(0.63-2.32)\end{array}$ & $\begin{array}{l}1.17 \\
(0.46-2.8)\end{array}$ & $\begin{array}{l}0.74 \\
(0.14-3.35)\end{array}$ & $\begin{array}{l}0.72 \\
(0.11-3.73)\end{array}$ & $\begin{array}{l}1.33 \\
(0.66-2.64)\end{array}$ & $\begin{array}{l}1.24 \\
(0.44-3.16)\end{array}$ & $\begin{array}{l}0.44 \\
(0.2-0.93)^{\dagger}\end{array}$ & $2.54(1.2-5.5)$ & $\begin{array}{l}2.76 \\
(0.89-9.39)\end{array}$ & NA \\
\hline $\begin{array}{l}\text { Alternative } \\
2 / 1 \text { vs } \\
\text { classical } 4 / 2 \\
\text { (pairwise } \\
\text { MA) }\end{array}$ & $\begin{array}{l}1.31 \\
(0.68-2.53)\end{array}$ & $\begin{array}{l}1.31 \\
(0.68-2.53)\end{array}$ & $\begin{array}{l}0.79 \\
(0.19-3.25)\end{array}$ & $\begin{array}{l}0.79 \\
(0.19-3.25)\end{array}$ & $1.47(0.72-3)$ & $1.47(0.72-3)$ & $\begin{array}{l}0.51 \\
(0.23-1.12)\end{array}$ & $\begin{array}{l}2.12 \\
(0.98-4.6)\end{array}$ & $\begin{array}{l}2.4 \\
(0.67-8.58)\end{array}$ & NA \\
\hline $\begin{array}{l}\text { Transitional } \\
2 / 1 \text { vs } \\
\text { classical } 4 / 2 \\
\text { (NMA) }\end{array}$ & $\begin{array}{l}0.79 \\
(0.32-1.86)\end{array}$ & $\begin{array}{l}0.81 \\
(0.26-2.46)\end{array}$ & $\begin{array}{l}1.03 \\
(0.19-5.27)\end{array}$ & $\begin{array}{l}1.01 \\
(0.15-6.42)\end{array}$ & $\begin{array}{l}0.72 \\
(0.26-1.88)\end{array}$ & $\begin{array}{l}0.77 \\
(0.23-2.71)\end{array}$ & $\begin{array}{l}0.57 \\
(0.25-1.29)\end{array}$ & $\begin{array}{l}2.07 \\
(0.96-4.42)\end{array}$ & $\begin{array}{l}2.14 \\
(0.67-7.22)\end{array}$ & NA \\
\hline $\begin{array}{l}\text { Transitional } \\
2 / 1 \text { vs } \\
\text { classical } 4 / 2 \\
\text { (pairwise } \\
\text { MA) }\end{array}$ & $\begin{array}{l}0.78 \\
(0.33-1.83)\end{array}$ & $\begin{array}{l}0.79 \\
(0.25-2.53)\end{array}$ & $\begin{array}{l}1.29 \\
(0.23-7.36)\end{array}$ & $\begin{array}{l}1.29 \\
([0.23-7.36)\end{array}$ & $\begin{array}{l}0.7 \\
(0.27-1.77)\end{array}$ & $\begin{array}{l}0.74 \\
(0.17-3.25)\end{array}$ & $\begin{array}{l}0.66 \\
(0.28-1.52)\end{array}$ & $\begin{array}{l}1.81 \\
(0.81-4.04)\end{array}$ & $\begin{array}{l}1.95 \\
(0.45-8.5)\end{array}$ & NA \\
\hline Sqrt(tau) & NA & 0.4266 & NA & 0.4657 & NA & 0.4924 & $\mathrm{~N} / \mathrm{A}$ & NA & 0.5932 & NA \\
\hline DIC & 47.24 & 47.8 & 32.22 & 32.62 & 46.91 & 46.78 & 37.91 & 40.51 & 39.11 & NA \\
\hline
\end{tabular}

†Statistically significant results.
The model with the smallest DIC is estimated to be the model that would best predict a replicate dataset that has the same structure as that currently observed. Tau is the common The model with the smallest $\mathrm{DIC}$ is estimated to be the model that would best predict
heterogeneity parameter, which indicates the variance of the underlying distribution.

Crl: Credible interval; DIC: Deviance information criterion; FE: Fixed-effects; MA: Meta-analysis; NA: Not applicable; NMA: Network meta-analysis; OR: Odds ratio; RE: Random-effects. 
Table 4. Summary of pairwise meta-analysis and network meta-analysis results: safety outcomes (anemia, diarrhea, fatigue, hand and foot syndrome).

\begin{tabular}{|c|c|c|c|c|c|c|c|c|c|}
\hline \multirow{3}{*}{$\begin{array}{l}\text { Outcome } \\
\text { Model }\end{array}$} & \multicolumn{2}{|c|}{ Anemia } & \multicolumn{2}{|c|}{ Diarrhea } & \multicolumn{2}{|c|}{ Fatigue } & \multicolumn{3}{|c|}{ Hand and foot syndrome } \\
\hline & \multicolumn{9}{|c|}{ Base case } \\
\hline & FE & RE1 & FE & RE1 & FE & RE1 & $\mathrm{FE}$ & RE1 & RE0.5 \\
\hline Comparisons & \multicolumn{9}{|l|}{ OR (95\% Crl) } \\
\hline $\begin{array}{l}\text { Alternative } \\
2 / 1 \text { vs classical } \\
4 / 2 \text { (NMA) }\end{array}$ & $0.86(0.29-2.4)$ & $\begin{array}{l}0.82 \\
(0.24-2.63)\end{array}$ & $0.95(0.35-2.3)$ & $\begin{array}{l}0.89 \\
(0.24-2.89)\end{array}$ & $\begin{array}{l}0.23 \\
(0.06-0.64)^{\dagger}\end{array}$ & $\begin{array}{l}0.24 \\
(0.06-0.76)^{\dagger}\end{array}$ & $\begin{array}{l}0.36 \\
(0.16-0.74)^{\dagger}\end{array}$ & $\begin{array}{l}0.33 \\
(0.12-0.79)^{\dagger}\end{array}$ & $0.35(0.15-0.77)^{\dagger}$ \\
\hline $\begin{array}{l}\text { Alternative } \\
2 / 1 \text { vs classical } \\
4 / 2 \text { (pairwise } \\
\text { MA) }\end{array}$ & $\begin{array}{l}0.97 \\
(0.33-2.89)\end{array}$ & $\begin{array}{l}0.97 \\
(0.33-2.89)\end{array}$ & $\begin{array}{l}1.27 \\
(0.52-3.07)\end{array}$ & $0.99(0.26-3.7)$ & $\begin{array}{l}0.51 \\
(0.18-1.41)\end{array}$ & $\begin{array}{l}0.47 \\
(0.14-1.57)\end{array}$ & $\begin{array}{l}0.44 \\
(0.21-0.92)^{\dagger}\end{array}$ & $\begin{array}{l}0.44 \\
(0.21-0.92)^{\dagger}\end{array}$ & $0.44(0.21-0.92)^{\dagger}$ \\
\hline $\begin{array}{l}\text { Transitional } \\
2 / 1 \text { vs classical } \\
4 / 2 \text { (NMA) }\end{array}$ & $\begin{array}{l}1.05 \\
(0.36-3.05)\end{array}$ & $\begin{array}{l}1.05 \\
(0.29-3.61)\end{array}$ & $\begin{array}{l}0.19 \\
(0.06-0.47)^{\dagger}\end{array}$ & $0.2(0.06-0.6)^{\dagger}$ & $\begin{array}{l}0.31 \\
(0.14-0.64)^{\dagger}\end{array}$ & $\begin{array}{l}0.32 \\
(0.12-0.8)^{\dagger}\end{array}$ & $\begin{array}{l}0.29 \\
(0.14-0.56)^{\dagger}\end{array}$ & $\begin{array}{l}0.26 \\
(0.1-0.59)^{\dagger}\end{array}$ & $0.27(0.12-0.57)^{\dagger}$ \\
\hline $\begin{array}{l}\text { Transitional } \\
2 / 1 \text { vs classical } \\
4 / 2 \text { (pairwise } \\
\text { MA) }\end{array}$ & $\begin{array}{l}0.92 \\
(0.27-3.12)\end{array}$ & $\begin{array}{l}0.92 \\
(0.27-3.12)\end{array}$ & $\begin{array}{l}0.32 \\
(0.12-0.87)^{\dagger}\end{array}$ & $\begin{array}{l}0.32 \\
(0.12-0.87)^{\dagger}\end{array}$ & $\begin{array}{l}0.34 \\
(0.15-0.75)^{\dagger}\end{array}$ & $\begin{array}{l}0.34 \\
(0.15-0.75)^{\dagger}\end{array}$ & $\begin{array}{l}0.37 \\
(0.18-0.75)^{\dagger}\end{array}$ & $\begin{array}{l}0.37 \\
(0.18-0.75)^{\dagger}\end{array}$ & $0.37(0.18-0.75)^{\dagger}$ \\
\hline Sqrt(tau) & NA & 0.4352 & NA & 0.6483 & NA & 0.5255 & NA & 0.4377 & 0.2566 \\
\hline DIC & 52.18 & 52.77 & 62.21 & 60.04 & 79.47 & 78.88 & 71.66 & 72.24 & 71.69 \\
\hline
\end{tabular}

† Statistically significant results.

The model with the smallest DIC is estimated to be the model that would best predict a replicate dataset that has the same structure as that currently observed. Tau is the common heterogeneity parameter, which indicates the variance of the underlying distribution.

Crl: Credible interval; DIC: Deviance information criterion; FE: Fixed-effects; MA: Meta-analysis; NA: Not applicable; NMA: Network meta-analysis; OR: Odds ratio; RE: Random-effect.

Table 5. Summary of pairwise meta-analysis and network meta-analysis results: safety outcomes (leukopenia, nausea, neutropenia, thrombocytopenia).

\begin{tabular}{|c|c|c|c|c|c|c|c|c|}
\hline \multirow{3}{*}{$\begin{array}{l}\text { Outcome } \\
\text { Model }\end{array}$} & \multicolumn{2}{|c|}{ Leukopenia } & \multicolumn{2}{|c|}{ Nausea } & \multicolumn{2}{|c|}{ Neutropenia } & \multicolumn{2}{|c|}{ Thrombocytopenia } \\
\hline & \multicolumn{8}{|c|}{ Base Case } \\
\hline & FE & RE & FE & RE & FE & RE & $\mathrm{FE}$ & RE \\
\hline Comparisons & \multicolumn{8}{|l|}{ OR (95\% Crl) } \\
\hline $\begin{array}{l}\text { Alternative } \\
2 / 1 \text { vs classical } \\
4 / 2 \text { (NMA) }\end{array}$ & $\begin{array}{l}0.94 \\
(0.02-38.94)\end{array}$ & $\begin{array}{l}0.94 \\
(0.02-39.57)\end{array}$ & $\begin{array}{l}1.41 \\
(0.04-12.57)\end{array}$ & $1.36(0.03-16.3)$ & $0.49(0.2-1.12)$ & $0.51(0.18-1.44)$ & $0.18(0.03-0.61)$ & $0.15(0.02-0.66)$ \\
\hline $\begin{array}{l}\text { Alternative } \\
2 / 1 \text { vs classical } \\
4 / 2 \text { (pairwise } \\
\text { MA) }\end{array}$ & NA & NA & NA & NA & $0.44(0.18-1.08)$ & $0.44(0.18-1.08)$ & $0.36(0.11-1.22)$ & $0.36(0.11-1.22)$ \\
\hline $\begin{array}{l}\text { Transitional } \\
2 / 1 \text { vs classical } \\
4 / 2 \text { (NMA) }\end{array}$ & $0.68(0.26-1.72)$ & $0.71(0.2-2.5)$ & $0.35(0.05-1.77)$ & $0.35(0.04-2.13)$ & $1.18(0.49-2.82)$ & $1.17(0.38-3.47)$ & $\begin{array}{l}0.47 \\
(0.26-0.83)^{\dagger}\end{array}$ & $0.46(0.19-1.01)$ \\
\hline $\begin{array}{l}\text { Transitional } \\
2 / 1 \text { vs classical } \\
4 / 2 \text { (pairwise } \\
\text { MA) }\end{array}$ & $0.6(0.21-1.72)$ & $0.72(0.13-4.11)$ & $0.4(0.07-2.1)$ & $0.4(0.07-2.1)$ & $1.36(0.53-3.49)$ & $1.36(0.53-3.49)$ & $0.54(0.3-0.97)^{\dagger}$ & $0.5(0.2-1.25)$ \\
\hline Sqrt(tau) & NA & 0.6312 & NA & 0.4751 & NA & 0.3915 & NA & 0.5547 \\
\hline DIC & 38.6 & 37.05 & 19.53 & 19.92 & 47.94 & 48.83 & 64.17 & 63.22 \\
\hline
\end{tabular}

† Statistically significant results.

The model with the smallest DIC is estimated to be the model that would best predict a replicate dataset that has the same structure as that currently observed. Tau is the common heterogeneity parameter, which indicates the variance of the underlying distribution.

Crl: Credible interval; DIC: Deviance information criterion; FE: Fixed-effects; MA: Meta-analysis; NA: Not applicable; NMA: Network meta-analysis; OR: Odds ratio; RE: Random-effects. 


\section{$M A$ results: heterogeneity diagnostics}

While no statistical heterogeneity was observed during the comparisons of the alternative $2 / 1$ schedule to the classical $4 / 2$ schedule, considerable statistical heterogeneity was found in the direct evidence related to the transitional 2/1 schedule when data were compared using classical pairwise MA for PFS $\left(I^{2}=95.3 \%\right)$. This result was based on two studies, and it could not be adjusted for the model due to a lack of individual patient-level data. This limits the generalizability of the meta-analytic average derived from the analysis.

\section{Exploratory NMA results}

Effectiveness

The alternative 2/1 and transitional 2/1 schedules demonstrated more favorable results compared with the classical $4 / 2$ schedule across all effectiveness outcomes. While there were no clear statistical differences between the regimens with regard to PFS, there was a tendency for the alternative $2 / 1$ and transitional $2 / 1$ schedules to prolong this outcome compared with the classical $4 / 2$ schedule. This trend continued for the alternative $2 / 1$ schedule in OS. The transitional 2/1 schedule had a statistical advantage over the classical 4/2 schedule. However, this finding should be interpreted with caution as it is based on the data from a single study.

Similar trends to those seen in the pairwise MA were observed in the NMA comparisons for CR, ORR, PD and SD for each treatment schedule comparison, with all having no statistical advantage. With regard to PD, a statistical advantage in prevention of disease progression was found for the alternative $2 / 1$ schedule over the classical 4/2 schedule. This finding was not statistically different within the pairwise MA; it should be interpreted with caution as the network was composed of only two studies.

Safety

No statistical differences between the dosing schedules were found for the NMA safety results, and rates of individual grade 3-4 AEs were variable across comparisons. Treatment with the classical 4/2 schedule vs the alternative 2/1 schedule was associated with numerically higher odds of anemia, diarrhea and neutropenia. The odds of leukopenia, nausea and thrombocytopenia associated with the classical $4 / 2$ schedule were numerically higher relative to the transitional 2/1 schedule.

\section{Potential Sources of bias \& their likely impact on the interpretation of the results}

Two broad groups of bias were identified among the included studies, and it is important to consider the impact of these while interpreting the results of the classical MA and the NMA. One inherent feature of all the studies' design - except one, the RESTORE trial [26] - was selection bias due to the uncontrolled, nonrandomized methods used to select the included sample. Although no formal risk-of-bias assessment was conducted for the included studies, those individual-study results reported for the transitional $2 / 1$ dosing schedule involved some form of patient channeling, which led to the very different results observed for patients who received that treatment. Two of the included studies [26,28] also potentially had some degree of 'immortal time bias' [36], given that those patients who transitioned would have been treated for longer, which could have made their results more favorable from the beginning of treatment.

\section{Discussion}

There is strong clinical evidence that demonstrates that sunitinib is effective in patients with mRCC when delivered at the conventional dose of $50 \mathrm{mg}$ daily with the classical schedule (4/2: 4 weeks yes and 2 weeks not). Although generally associated with acceptable, and manageable toxicity, sunitinib frequently results in AEs that could potentially lead to early treatment discontinuation. Recent literature suggests there may be alternative dosing schedules that maintain the established efficacy profile seen with the classical $4 / 2$ schedule while also minimizing toxicity [17-20,26,28,31,35].

Schedule changes with the classical schedule may be considered before lowering the dose to retain the optimal level of exposure to sunitinib. However, there is currently some doubts within the existing literature on which schedule may be optimal with regards to safety and effectiveness for the treatment of mRCC. An SLR and MA were conducted to compare two alternative sunitinib dosing schedules, the alternative $2 / 1$ and the transitional $2 / 1$ schedule, to the classical $4 / 2$ schedule.

The comparison of sunitinib dosing schedules within the existing literature is limited and direct comparisons are not available, especially for the transitional 2/1 schedule. A systematic review summarizing sunitinib dosing 
schedules was conducted in 2014 [37]. Yet, the current study includes updated literature and also has meta-analyzed the outcomes reported in included relevant studies. Therefore, it is a comprehensive overview of evidence and is reflective of current real-world clinical practice. It is the first peer-reviewed study to our knowledge to systematically evaluate the comparative safety and effectiveness of sunitinib dosing schedules in mRCC.

A pairwise MA found the alternative $2 / 1$ and transitional $2 / 1$ schedules to numerically prolong PFS when compared with the classical $4 / 2$ schedule. Nevertheless, only the alternative $2 / 1$ comparison had a statistical advantage, and this difference was not evident in sensitivity analyses.

The PFS estimate generated for the NMA compared the transitional 2/1 to the classical 4/2 schedule indicated that the former schedule has a statistical advantage in extending PFS compared with the latter; however, the direct comparison did not find any such statistical advantage. The divergence may have resulted from the lack of sufficient power to detect differences due to the sample size disparity in the studies by Bracarda et al. [28] and Pan et al. [31] ( $N=460$ and $N=108$, respectively). Nonetheless, we were unable to fully account for the inconsistencies observed for these two studies due to a lack of publicly available data.

OS was also numerically prolonged for the alternative $2 / 1$ and transitional $2 / 1$ schedules compared with the classical $4 / 2$ schedule. Only the comparison with transitional $2 / 1$ was statistically different for OS, but this result should be viewed with caution as it is based on data from only one study [28]. Within this study, patients in the transitional 2/1 arm were treated for a longer duration than the classical 4/2 patients, which indicated that they likely would have lived longer irrespective of the effectiveness of the former schedule.

Hypertension and thyroid dysfunction was captured in most of the included studies but were however not analyzed in this analysis as the safety outcomes for which MA and NMA were conducted were considered more clinically relevant to sunitinib treatment. Among safety outcomes, the most robust data within the MA related to the occurrence of hand-foot syndrome. The alternative $2 / 1$ and transitional $2 / 1$ schedules demonstrated statistically lower odds of the disease than the classical $4 / 2$ schedule in both pairwise MA and NMA results. Sunitinib has also been found to have a better outcome in randomized settings in this safety outcome even in comparison to other VEGFR inhibitors, such as cabozantinib [38]. Future research should compare the impact of VEGFR inhibitors on this and other safety outcomes. Further advantages were seen with the transitional $2 / 1$ schedule in terms of fatigue and diarrhea.

A key strength of the MAs in our study was the generation of summary statistics and assessment of statistical heterogeneity using well-established methods. While the estimates for most outcomes did not show clear statistical advantages, these findings may be important in clinical practice.

Our analysis has some limitations. Of the 11 studies included in the review, only one was an RCT, and the remaining ten were observational in design. An MA of clinical data derived from an observational design is uncommon and challenging in terms of bias and in finding consistency in reported outcomes and definitions. Some of the treatment arms included small sample sizes, which raised the possibility that the studies may have had insufficient power to detect differences in the outcomes measured. In addition, of the two potentially relevant prospectively conducted studies identified by the review, only the RESTORE trial conducted by Lee et al. [26] provided comparative data, while the other study by Jonasch et al. [39] was excluded from the review for lack of a comparative arm.

There were several limitations in the statistical analysis of PFS outcomes within the study set. First, PFS data were not available in the RCT publication, so TTP data were used as a proxy. While PFS is preferred over TTP in oncology, it appears they could be used interchangeably on the assumption that deaths occurred in studies reported in the TTP outcome are not related to the cancer [40]. We were, however, unable to determine whether this was the case for the RCT [26] in reporting this outcome because of our lack of access to patient-level data. Further, considerable statistical heterogeneity $\left(I^{2}=95.3 \%\right)$ was found in the direct evidence related to the transitional $2 / 1$ schedule, but this result was based on only two studies that had conflicting outcomes. Selection bias, differences in sample sizes and the possible impact of confounding resulting from the use of observational data are likely explanations for this difference in observed effects for the same intervention across the studies.

The inclusion of arms for the transitional $2 / 1$ schedule in this review may have also opened the analysis to additional immortal time bias. However, an examination of the studies reporting data on time to switching from the classical $4 / 2$ schedule to the alternative $2 / 1$ schedule found that the switch occurs after approximately 3 months [28,31]. It is therefore less likely that immortal time bias truly existed in the studies evaluating transitional $2 / 1$ schedules. 
Further, there was a lack of adjusted observational data within the study set, and that prevented potential confounding variables from being identified. The analysis could have benefited from conducting propensity score matching of included studies for balancing comparison arms with respect to known and unknown prognostic factors, but this is only achievable with patient-level data, to which we did not have access for any of the studies. Two publications, however, reported numerical data that had been adjusted for confounding factors using Cox proportional hazards regression analysis $[29,35]$. There was also a limited number of studies evaluating or providing data for comparisons. This prevented us from estimating a random-effects variance. Inferences from these outcomes are based on results of the fixed-effects models, which do not take the heterogeneity among studies into account.

The SURF study (ClinicalTrials.gov: NCT02689167), a randomized, Phase II trial, will further explore the benefits of using a transitional sunitinib schedule versus applying dose reduction. It is an ongoing investigation of the safety and efficacy of changing dosing schedules versus decreasing the dose of sunitinib in patients with mRCC experiencing toxicity on the $4 / 2$ schedule. The primary end point of the study, which is expected to enroll 248 patients, is to determine the duration of treatment in both arms [41].

\section{Conclusion}

Evidence from this systematic review and MA indicates the benefits of sunitinib dosing schedules on clinical outcomes among patients with mRCC. The results show that the alternative $2 / 1$ and transitional $2 / 1$ schedules were generally better tolerated and more effective than the classical $4 / 2$ schedule. While the estimates for most outcomes were not statistically significant, favorable trends were observed and therefore, the findings from these MAs could be useful in determining which schedules patients can potentially be offered in real-world clinical practice. However, these results should be interpreted with caution due to the heterogeneity between the studies and some lack of consistency in reporting of outcomes.

The trends observed in the present study favoring alternative and transitional sunitinib schedules are in accordance with routine real-world clinical practice. Guidelines recommend treating mRCC with the $50 \mathrm{mg}$ daily classical $4 / 2$ schedule. Patients who cannot tolerate sunitinib at the classical $4 / 2$ schedule may be moved to the $2 / 1$ schedule to retain the daily $50 \mathrm{mg}$ dosing when possible. Thus, our findings are aligned with current real-world practice. Despite that this research synthesis provides the best available evidence to date on the subject, the evidence base for studies including the transitional $2 / 1$ schedule is weak and sparse. Future prospective trials or real-world evidence evaluating this schedule will be helpful in determining the relevance of this approach for treating patients with mRCC.

\section{Future perspective}

Providing patient-centred care using a personalized approach is playing a key role in the management of mRCC and oncology in general. This study shows that the evidence to support the use of a treatment in real world clinical practice may evolve over time to provide better outcomes and improved tolerability.

\section{Acknowledgments}

The authors thank R Hughes and I Iheanacho of Evidera, who provided medical writing and editorial support during the manuscript preparation. We will also like to thank J Kenny and O Arisa who assisted with the review of the literature.

\section{Financial \& competing interests disclosure}

This work was supported by Pfizer Inc. No grant number is applicable. S Abogunrin and K Fahrbach are employees of Evidera, a research and consulting firm to the biopharmaceutical industry. As salaried employees of Evidera, they do not accept personal remuneration of any type from supporting organizations. AO Ashaye was an employee of Evidera and did not receive any personal remuneration from the supporting organization. JC Cappelleri, G Zanotti, K Ramaswamy, D Thomaidou, AG Clair and L Serfass are employees and stockholders of Pfizer Inc. S Srinivas has no conflict of interest. This study was designed by researchers employed or commissioned by the sponsor. All authors employed or commissioned by the sponsor participated in the interpretation of the data a preparation of the manuscript. The authors have no other relevant affiliations or financial involvement with any organization or entity with a financial interest in or financial conflict with the subject matter or materials discussed in the manuscript apart from those disclosed.

The manuscript was developed with editorial support from researchers commissioned by the sponsor. All authors had full access to the data, and the corresponding author had final responsibility for the decision to submit for publication. 
Executive Summary

Background

- Sunitinib, a tyrosine kinase inhibitor (TKI), is a first-line treatment that has demonstrated improved outcomes in metastatic renal cell carcinoma (mRCC).

- The optimal dosing schedule to maintain its effectiveness - while reducing toxicity - remains an important clinical question.

- The objective of this review was to compare the effectiveness and safety of different sunitinib dosing schedules for mRCC treatment.

Methods

- A systematic review and meta-analysis of randomized controlled trials and longitudinal observational studies, published in English, were conducted to assess clinical results of the classical 4/2, alternative 2/1 and transitional 2/1 sunitinib schedules for treating patients with mRCC.

- Bayesian mixed treatment comparison analyses and network meta-analyses were performed to compare the relative treatment effects of schedules on overall survival (OS), progression-free survival (PFS), response and grade 3-4 adverse events (AEs).

Results

- Of the 692 records reviewed, 11 primary studies (one randomized trial and ten observational studies) were included and nine were considered eligible for the meta-analysis.

- Treatment with the alternative $2 / 1$ schedule reduced the risk of disease progression or death by $25 \%$ compared with the classical 4/2 schedule (hazard ratio [HR]: 0.75 [95\% credible interval (Crl): 0.60, 0.94]).

- A numerical but not 'statistical' benefit in PFS was observed with the transitional compared with the classical schedule.

- The odds of fatigue and diarrhea were lower for the transitional 2/1 schedule compared with the classical 4/2 schedule (odds ratio [OR]: 0.32 [95\% Crl: 0.12-0.87]).

- The alternative 2/1 (OR: 0.44 [95\% Crl: 0.21-0.92]) and transitional 2/1 (OR: 0.37 [95\% Crl: 0.18-0.75]) schedules demonstrated statistically lower odds of hand and foot syndrome than the classical 4/2 schedule.

Conclusion

- Benefits of alternative sunitinib schedules on clinical outcomes among patients with mRCC were demonstrated.

- The alternative $2 / 1$ and transitional $2 / 1$ schedules appeared generally better tolerated and more effective than the classical $4 / 2$ schedule.

Data sharing statement

Upon request, and subject to certain criteria, conditions and exceptions (see www.pfizer.com/science/clinical-trials/trial-data-andresults for more information), Pfizer will provide access to individual de-identified participant data from Pfizer-sponsored global interventional clinical studies conducted for medicines, vaccines and medical devices (1) for indications that have been approved in the US and/or EU or (2) in programs that have been terminated (i.e., development for all indications has been discontinued). Pfizer will also consider requests for the protocol, data dictionary and statistical analysis plan. Data may be requested from Pfizer trials 24 months after study completion. The de-identified participant data will be made available to researchers whose proposals meet the research criteria and other conditions, and for which an exception does not apply, via a secure portal. To gain access, data requestors must enter into a data access agreement with Pfizer.

Open access

This work is licensed under the Attribution-NonCommercial-NoDerivatives 4.0 Unported License. To view a copy of this license, visit http://creativecommons.org/licenses/by-nc-nd/4.0/

\section{References}

1 Ljungberg B, Campbell SC, Choi HY et al. The epidemiology of renal cell carcinoma. Eur Urol. 60(4), 615-621 (2011).

2 Ferlay J, Shin HR, Bray F, Forman D, Mathers C, Parkin DM. GLOBOCAN Cancer Incidence and Mortality Worldwide. 2012; http://globocan.iarc.fr.

3 Gupta K, Miller JD, Li JZ, Russell MW, Charbonneau C. Epidemiologic and socioeconomic burden of metastatic renal cell carcinoma (mRCC): a literature review. Cancer Treat. Rev. 34(3), 193-205 (2008).

4 Ljungberg B, Cowan NC, Hanbury DC et al. EAU guidelines on renal cell carcinoma: the 2010 update. Eur. Urol. 58(3), 398-406 (2010).

5 Gangadaran SGD. Current management options in metastatic renal cell cancer. Oncol. Rev. 11(2), 339 (2017). 
6 Hammers HJ, Plimack ER, Infante JR et al. Safety and efficacy of nivolumab in combination with ipilimumab in metastatic renal cell carcinoma: the CheckMate 016 study. J. Clin. Oncol. 35(34), 3851-3858 (2017).

7 Motzer RJ, Escudier B, McDermott DF et al. Nivolumab versus everolimus in advanced renal-cell carcinoma. N. Engl. J. Med. 373(19), 1803-1813 (2015).

8 Motzer RJ, Tannir NM, McDermott DF et al. Nivolumab plus ipilimumab versus sunitinib in advanced renal-cell carcinoma. $N$. Engl. J. Med. 378(14), 1277-1290 (2018).

9 Ono Pharmaceutical, Bristol-Myers Squibb. Bristol-Myers Squibb receives FDA approval for opdivo (nivolumab) in previously treated locally advanced or metastatic urothelial carcinoma, a type of bladder cancer. 2017, 14.

10 Motzer RJ, Hutson TE, Tomczak P et al. Overall survival and updated results for sunitinib compared with interferon alfa in patients with metastatic renal cell carcinoma. J. Clin. Oncol. 27(22), 3584-3590 (2009).

11 Motzer RJ, Hutson TE, Tomczak P et al. Sunitinib versus interferon alfa in metastatic renal-cell carcinoma. N. Engl. J. Med. 356(2), $115-124$ (2007).

12 Escudier B, Grünwald V, Gillessen S et al. Renal cell carcinoma: ESMO Clinical Practice Guidelines for diagnosis, treatment and follow-up. 2019.

13 Gore M, Szczylik C, Porta C et al. Final results from the large sunitinib global expanded-access trial in metastatic renal cell carcinoma. Br. J. Cancer 113(1), 12 (2015).

14 Motzer RJ, Hutson TE, Cella D et al. Pazopanib versus sunitinib in metastatic renal-cell carcinoma. N. Engl. J. Med. 369(8), 722-731 (2013).

15 Vrdoljak E, Géczi L, Mardiak J et al. Central and Eastern European experience with sunitinib in metastatic renal cell carcinoma: a sub-analysis of the global expanded-access trial. Pathol. Oncol. Res. 21(3),775-782 (2015).

16 Houk BE, Bello CL, Poland B, Rosen LS, Demetri GD, Motzer RJ. Relationship between exposure to sunitinib and efficacy and tolerability endpoints in patients with cancer: results of a pharmacokinetic/pharmacodynamic meta-analysis. Cancer Chemother. Pharmacol. 66(2), 357-371 (2010).

17 Atkinson BJ, Kalra S, Wang X et al. Clinical outcomes for patients with metastatic renal cell carcinoma treated with alternative sunitinib schedules. J. Urol. 191(3), 611-618 (2014).

18 Barrios $\mathrm{CH}$, Hernandez-Barajas D, Brown MP et al. Phase II trial of continuous once-daily dosing of sunitinib as first-line treatment in patients with metastatic renal cell carcinoma. Cancer 118(5), 1252-1259 (2012).

19 Buti S, Donini M, Bersanelli M, Gattara A, Leonardi F, Passalacqua R. Feasibility, safety, and efficacy of an alternative schedule of sunitinib for the treatment of patients with metastatic renal cell carcinoma: a retrospective study. Drugs R $\mho D$ 17(4), 585-596 (2017).

20 Ohba K, Miyata Y, Yasuda T et al. Efficacy and safety of sunitinib alternate day regimen in patients with metastatic renal cell carcinoma in Japan: Comparison with standard 4/2 schedule. Asia Pac. J. Clin. Oncol. 14(3), 153-158 (2018).

21 Hutton B, Salanti G, Caldwell DM et al. The PRISMA extension statement for reporting of systematic reviews incorporating network meta-analyses of health care interventions: checklist and explanations. Ann. Intern. Med. 162(11), 777-784 (2015).

22 Lobo FS, Wagner S, Gross CR, Schommer JC. Addressing the issue of channeling bias in observational studies with propensity scores analysis. Res. Social Adm. Pharm. 2(1), 143-151 (2006).

23 Caldwell DM, Ades AE, Higgins JP. Simultaneous comparison of multiple treatments: combining direct and indirect evidence. BMJ 331(7521), 897-900 (2005).

$24 \mathrm{Lu} \mathrm{G}$, Ades AE. Combination of direct and indirect evidence in mixed treatment comparisons. Stat. Med. 23(20), 3105-3124 (2004).

25 Higgins JP, Thompson SG. Quantifying heterogeneity in a meta-analysis. Stat. Med. 21(11), 1539-1558 (2002).

26 Lee JL, Kim MK, Park I et al. RandomizEd Phase II trial of Sunitinib four weeks on and two weeks off versus Two weeks on and One week off in metastatic clear-cell type REnal cell carcinoma: RESTORE trial. Ann. Oncol. 26(11), 2300-2305 (2015).

27 Neri B, Vannini A, Brugia $M$ et al. Biweekly sunitinib regimen reduces toxicity and retains efficacy in metastatic renal cell carcinoma: a single-center experience with 31 patients. Int. J. Urol. 20(5), 478-483 (2013).

28 Bracarda S, Iacovelli R, Boni $\mathrm{L}$ et al. Sunitinib administered on $2 / 1$ schedule in patients with metastatic renal cell carcinoma: the RAINBOW analysis. Ann. Oncol. 26(10), 2107-2113 (2015).

29 Kondo T, Takagi T, Kobayashi H et al. Superior tolerability of altered dosing schedule of sunitinib with 2-weeks-on and 1-week-off in patients with metastatic renal cell carcinoma-comparison to standard dosing schedule of 4-weeks-on and 2-weeks-off. Jpn J. Clin. Oncol. 44(3), 270-277 (2014).

30 Najjar YG, Mittal K, Elson P et al. A 2 weeks on and 1 week off schedule of sunitinib is associated with decreased toxicity in metastatic renal cell carcinoma. Eur. J. Cancer 50(6), 1084-1089 (2014).

31 Pan X, Huang H, Huang Y et al. Sunitinib dosing schedule 2/1 improves tolerability, efficacy, and health-related quality of life in Chinese patients with metastatic renal cell carcinoma. Urol. Oncol. 33(6), 268 e269-215 (2015).

32 Miyake H, Harada K, Miyazaki A, Fujisawa M. Improved health-related quality of life of patients with metastatic renal cell carcinoma treated with a 2 weeks on and 1 week off schedule of sunitinib. Med. Oncol. 32(3), 78 (2015). 
33 Cheng W, Kletas V, De Lemos ML, Man S, Kollmannsberger CK. Survival Outcomes Associated with Different Sunitinib Dosing Regimens in Metastatic Renal Cell Carcinoma. ASCO Annual Meeting, Chicago, IL (2014).

34 Ezz El Din M. Sunitinib 4/2 versus 2/1 schedule for patients with metastatic renal cell carcinoma: tertiary care hospital experience. Clin. Genitourin. Cancer 15(3), e455-e462 (2017).

35 Suo A, Iqbal U, Lim J et al. Outcomes and drug costs of sunitinib regimens for metastatic renal cell carcinoma: a provincial population-based study. Clin. Genitourin. Cancer 15(3), e397-e404 (2017).

36 Suissa S. Immortal time bias in pharmaco-epidemiology. Am. J. Epidemiol. 167(4), 492-499 (2008).

37 Guida FM, Santoni M, Conti A et al. Alternative dosing schedules for sunitinib as a treatment of patients with metastatic renal cell carcinoma. Crit. Rev. Oncol. Hematol. 92(3), 208-217 (2014).

38 Choueiri TK, Halabi S, Sanford BL et al. Cabozantinib versus sunitinib as initial targeted therapy for patients with metastatic renal cell carcinoma of poor or intermediate risk: the alliance A031203 CABOSUN trial. J. Clin. Oncol. 35(6), 591-597 (2017).

39 Jonasch E, Slack RS, Geynisman DM et al. Phase II study of two weeks on, one week off sunitinib scheduling in patients with metastatic renal cell carcinoma. J. Clin. Oncol. 36(16), 1588-1593 (2018).

40 Soria JC, Massard C, Le Chevalier T. Should progression-free survival be the primary measure of efficacy for advanced NSCLC therapy? Ann. Oncol. 21(12), 2324-2332 (2010).

41 Mouillet G, Paillard MJ, Maurina T et al. Open-label, randomized multicentre Phase II study to assess the efficacy and tolerability of sunitinib by dose administration regimen (dose modification or dose interruptions) in patients with advanced or metastatic renal cell carcinoma: study protocol of the SURF trial. Trials 19(1), 221 (2018). 